Reprod. Nutr. Dévelop., 1981, 21 (1), 1-17.

\title{
Variations au cours de la journée des teneurs en certains constituants plasmatiques chez la jument poulinière
}

\author{
par M. DOREAU, W. MARTIN-ROSSET *, J.-P. BARLET ** \\ avec la collaboration technique de H. DUBREUCQ *, Renée LEFAIVRE, R. ROUX ** \\ ef Claire SORNET \\ Laboratoire de la Production laitière, INRA \\ * Laboratoire de la Production de Viande, INRA \\ ** Laboratoire des Maladies métaboliques, INRA \\ Theix, 63110 Beaumont, France.
}

Summary. Circadian variations in the concentrations of some plasma components in the heavy brood mare.

Twenty-one circadian blood sample profiles were made in heavy brood mares during pregnancy, lactation or the dry, non-pregnant period. Thc mares were fed forage-rich diets containing different levels of energy and nitrogen (table 1).

Each profile consisted of 7 samples taken at 8 a.m., 11 a.m., 3 p.m., 7 p.m., 11 p.m., $4 \mathrm{a} . \mathrm{m}$. and $8 \mathrm{a} . \mathrm{m}$. The animals were fed at 8:30 a.m. The eleven plasma components evaluated were glucose, non-esterified fatty acids, $\beta$-hydroxybutyrate, acetate, alanine, insulin (energy metabolites), urea, total protein (nitrogen metabolites), calcium, inorganic phosphorus and magnesium (mineral metabolites). Profile results are shown in table 2 and figure 1.

Glucose, insulin and alanine increased and non-esterified fatty acids, acetate and $\beta$-hydroxybutyrate decreased during the prandial period (between $8 \mathrm{a} . \mathrm{m}$. and $11 \mathrm{a} . \mathrm{m}$.). The glucose and insulin peaks were short, while the other components returned slowly to preprandial values. Acetate and $\beta$-hydroxybutyrate were maximal during the night, while urea increased moderately during the prandial period. Protein concentration did not vary. The effect of feeding on minerals was moderate : there was a slight increase in calcium and magnesium and a decrease in phosphorus.

The following differences were related to diet or physiological state (figs. $2,3,4$ ) :

- higher glucose and insulin peaks when concentrate intake was high ;

- lower circadian variation of non-esterified fatty acids, $\beta$-hydroxybutyrate and acetate in dry, non-pregnant, well-fed mares ;

- higher acetate levels with a high-forage dief ;

- more rapid alanine decrease during late pregnancy than in early lactation ;

- higher circadian variations in minerals during lactation than during pregnancy.

The prandial variations of the following factors were significantly correlated (table 3 ):

- glucose and insulin,

- acetate and $\beta$-hydroxybutyrate,

- $\beta$-hydroxybutyrate and non-esterified fatty acids,

- acetate and non-esterified fatty acids.

The origin of these variations is discussed. Two factors intervened :

1) exogenous production Very rapid digestion of the concentrate induced an increase in glucose (and thus in insulin) and alanine. The volatile fatty acids produced in the hindgut caused nocturnal peaks of acetate and $\beta$-hydroxybutyrate ; 
2) a lipogenic phase (which began after mean meal intake) alternating with a lipolytic phase, providing constant coverage of the energy needs This explains the large postprandial decreases in non-esterified fatty acids, acetate and $\beta$-hydroxybutyrate.

Les teneurs en certains constituants plasmatiques de la circulation périphérique fournissent un moyen simple de caractériser l'étał nutritionnel chez différentes espèces animales. Elles sont cependant susceptibles de larges variations au cours du nycthémère, dues à la cinétique de la digestion et à des modifications d'ordre métabolique. L'utilisation de critères plasmatiques chez le cheval nécessite donc de connaître ces variations. Chez les Equidés, l'évolution de la glycémie, présentant un pic postprandial plus ou moins marqué, a été suivie par de nombreux auteurs, donł Takagi ef Sakurai (1971), Gill, Skwarlo et Flisinska-Bojanowska (1974) et Evans, Thompson et Winget (1974). Ces derniers ont en outre mis en évidence une évolution parallèle de l'insulinémie. Errington (1937) a montré une diminution post-prandiale de la phosphatémie, mais pas de variation de la calcémie. Différents autres constituants n'ont fait l'objet que d'études partielles : le repas provoque une diminution de la teneur en acides gras non estérifiés (Krzywanek, 1969 ; Anderson, 1975) ef une augmentation de la teneur en acides aminés libres (Johnson et Hart, 1974). La fotalité de ces travaux a été réalisée sur chevaux de selle à l'entretien ou au travail.

Cette étude a pour objet de mesurer sur des juments de races lourdes, à besoins élevés, les variations au cours du nycthémère de différents constituants plasmatiques témoins du métabolisme énergétique (glucose, insuline, acides gras non estérifiés, $\beta$-hydroxybutyrate, acétate, alanine), azoté (protéines, urée) ou minéral (calcium, phosphore, magnésium). L'objectif était triple : relier l'évolution des constituants étudiés aux connaissances acquises sur la nature et l'importance des produits terminaux de la digestion et confirmer leurs inter-relations métaboliques, mettre en évidence l'effet éventuel du type de régime et de l'état physiologique, préciser l'heure optimale de prélèvement sanguin pour des études ultérieures à caractère nutritionnel.

\section{Matériel et méthodes.}

Les cinétiques de prélèvements sanguins ont été réalisées dans deux essais d'alimentation en fin de gestation et début de lactation, dont les résultats seront exploités par ailleurs (Martin-Rosset et Doreau, non publié). Le premier élait destiné à estimer les variations du niveau d'ingestion volontaire aux environs du poulinage ; le second à déterminer le niveau minimum d'apport énergétique compatible avec des résultats zootechniques satisfaisants.

Animaux ef régimes. - Les animaux utilisés étaient des juments âgées de 5 à 11 ans, appartenant à différentes races lourdes françaises, et pesant entre 635 et $860 \mathrm{~kg}$ au lendemain de la mise-bas. Ces juments étaient soit gestantes puis allaitantes, soit vides.

Les régimes étaient très différents entre les deux essais : la proportion d'aliment concentré était voisine de 10 p. 100 dans le premier et de 20 p. 100 dans le deuxième ; 


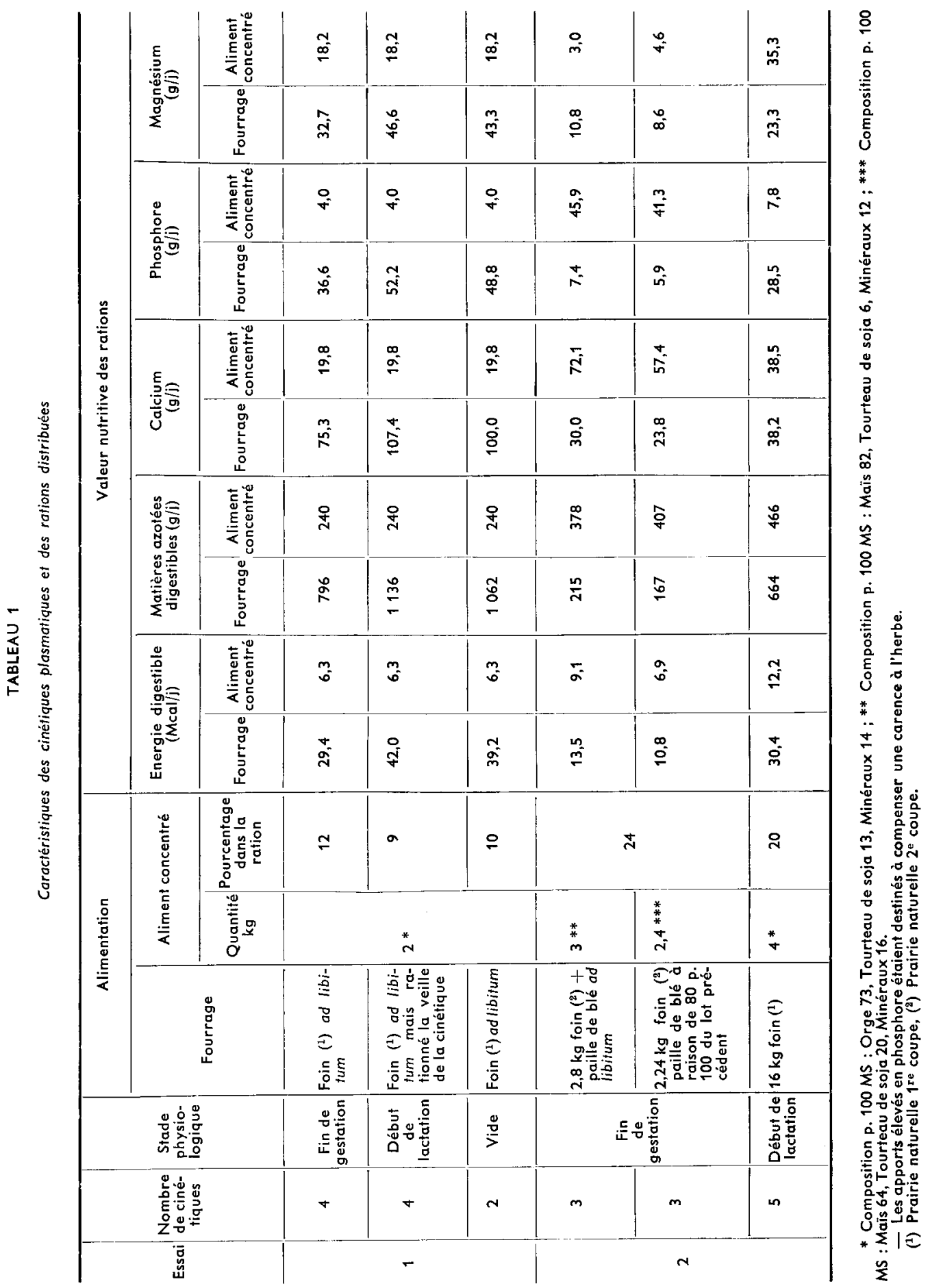


l'apport énergétique et azoté global était cependant plus élevé lors du premier essai. Au cours d'un même essai, les apports énergétiques et azotés étaient beaucoup plus élevés en lactation qu'en gestation.

L'essai 1 a été effectué sur 4 juments gestantes puis allaitantes et 2 vides recevant $2 \mathrm{~kg}$ d'aliment concentré et du foin ad libitum (tabl. 1). Les juments étaient conduites en stabulation libre par lots de 2 ; les aliments étaient distribués à $8 \mathrm{~h} 30$, le concentré individuellement et le fourrage par lot.

Dans l'essai 2, 6 juments ont été réparties par couples en deux groupes de poids vif et d'appétit voisins alimentés en fin de gestation selon deux niveaux énergétiques différents, mais à même niveau azoté, et recevant une ration commune en début de lactation. Une jument du groupe à bas niveau énergétique ayant avorté, 5 animaux ont été utilisés en lactation. Les juments étaient alimentées individuellement en stalles et recevaient le foin, l'aliment concentré et la moitié de la paille à 8 h 30 , le complément de paille d̀ $16 \mathrm{~h} 30$.

Prélèvements sanguins. -10 cinétiques ont été effectuées lors du 1 er essai ( 4 juments en fin de gestation, les mêmes en début de lactation et 2 juments vides) et 11 lors du $2^{\mathrm{e}}$ essai ( 6 juments en fin de gestation et 5 d'entre elles en début de lactation). Chaque cinétique a comporté 7 prises de sang à $8,11,15,19,23,4$ ef $8 \mathrm{~h}$. Elles ont eu lieu au printemps, pour les deux essais respectivement 14 à 24 et 31 à 61 jours avant la mise-bas, 13 à 20 et 7 à 17 jours après la mise-bas.

Les prises de sang ont été effectuées au niveau de la veine jugulaire. Le sang, recueilli sur héparine, était aussitôt centrifugé. Une partie du plasma était déprotéinisée à l'acide perchlorique $0,6 \mathrm{M}$ puis filtrée ; les tubes de plasma déprotéinisé ou non étaient conservés à $-15^{\circ} \mathrm{C}$ avant analyses.

Méthodes d'analyse. - Le glucose a été dosé par la gluco-oxydase (Trinder, 1969), l'urée par la méthode à la diacétylmonoxime (Moore et Sax, 1965), les protéines par la méthode du biuret, les acides gras non estérifiés par colorimétrie d'après Rémésy et Demigné (1976), le $\beta$-hydroxybutyrate par méthode enzymatique selon Williamson et Mellanby (1974), l'acétate par méthode enzymatique selon Guynn et Veech (1975), I'insuline par radio-immunologie selon la méthode du double anticorps (Hales et Randle, 1963), le calcium et le magnésium par spectrophotométrie d'absorption afomique, le phosphore minéral par colorimétrie. L'alanine a été dosée par méthode enzymatique uniquement sur les cinétiques de l'essai 2 , selon la méthode de Williamson (1974).

Expression des résultats. - Les constituants étudiés sont regroupés en classes, selon qu'ils sont liés au métabolisme énergétique, azoté ou minéral. Deux facteurs de variation sont distingués : 10 le régime alimentaire, en comparant les régimes du $1^{\mathrm{er}}$ et du $2^{\mathrm{e}}$ essai ; $2^{\circ}$ le stade physiologique, en comparant les animaux en gestation, en lactation et vides. Il est cependant probable que l'effet de l'état physiologique ait inferféré avec celui du régime ou du niveau des apports énergétiques, azotés et minéraux. En effet, la valeur énergétique des aliments et les besoins de la jument, en particulier en lactation, sont mal connus ; il est donc difficile de connaître le taux de couverture des besoins par les apports alimentaires. 
L'évolution des différents constituants au cours de la journée a été reliée aux activités alimentaires. Bien que les heures et durées des prises alimentaires n'aient pas été relevées, les études du comportement alimentaire du cheval à l'auge (Ruckebusch, Vigroux ef Candau, 1976 : Doreau, 1978) permettent de définir un schéma des activités alimentaires approximatif, mais probable : les juments ingèrent à partir de $8 \mathrm{~h} 30$ l'aliment concentré à raison de 10 à $15 \mathrm{~min}$ par $\mathrm{kg}$ de matière sèche. Immédiatement après, elles effectuent un repas principal de fourrage de durée généralement

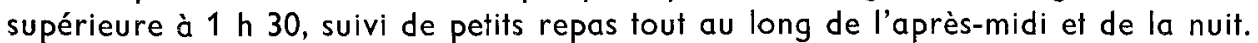
Une reprise d'ingestion se manifeste vers $5 \mathrm{~h}$ du matin, lorsque le jour se lève. Pour les animaux rationnés, le premier repas de fourrage après la distribution a dû être plus important ; les animaux ayant reçu 2 distributions de paille ont dû effectuer un deuxième grand repas après la distribution de l'après-midi.

\section{Résultats.}

Cinétiques moyennes (łabl. 2, fig. 1). - Entre les prélèvements de $8 \mathrm{~h}$ (avant la distribution d'aliments), et de $11 \mathrm{~h}$ la concentration de tous les constituants liés au métabolisme énergétique varie très fortement : les teneurs en glucose, alanine et insuline sont respectivement multipliées par 2, 2 et 7 environ, les teneurs en acides gras non estérifiés, le $\beta$-hydroxybutyrate et l'acétate diminuent respectivement de 54,36 et 36 p. 100 . A 15 h, soit 6 h 30 après la distribution d'aliments, la glycémie est presque revenue à la valeur pré-prandiale; elle diminue très progressivement jusqu'au lendemain matin. L'insulinémie diminue plus lentement : elle revient à la valeur préprandiale à $19 \mathrm{~h}$ et ne varie plus jusqu'au lendemain matin. L'alanine et les acides gras non estérifiés présentent respectivement un maximum et un minimum de concentration à $15 \mathrm{~h}$, puis reviennent progressivement aux valeurs pré-prandiales. Le $\beta$-hydroxybutyrate ef l'acétate augmentent à partir de $11 \mathrm{~h}$ en présentant un pic de concentration dans la nuit, à $23 \mathrm{~h}$ pour l'acétate ef à $4 \mathrm{~h}$ pour le $\beta$-hydroxybutyrate.

TABLEAU 2

Concentrations moyennes et limites en différents constituants plasmatiques au cours de la journée

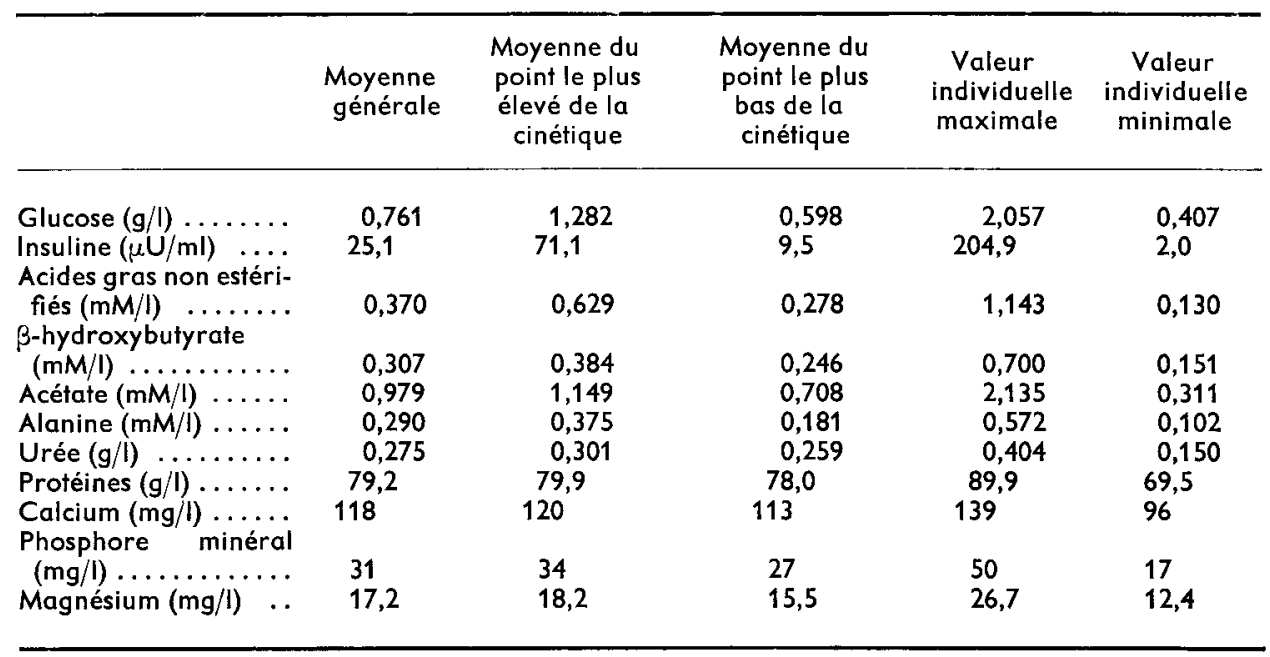


La teneur en urée augmente faiblement, de 7 p. 100 en moyenne, mais systématiquement, entre 8 et $11 \mathrm{~h}$. Elle diminue progressivement jusqu'à $4 \mathrm{~h}$ et augmente faiblement à $8 \mathrm{~h}$. La teneur moyenne en protéines varie très peu au cours de la journée, de $2,4 \mathrm{p} .100$, entre un minimum à $8 \mathrm{~h}$ ef un maximum à $18 \mathrm{~h}$, mais les teneurs individuelles sont beaucoup plus variables, l'écart entre minimum ef maximum pouvant atteindre $1 \mathrm{~g} / 100 \mathrm{ml}$, soit $12 \mathrm{p} .100$.
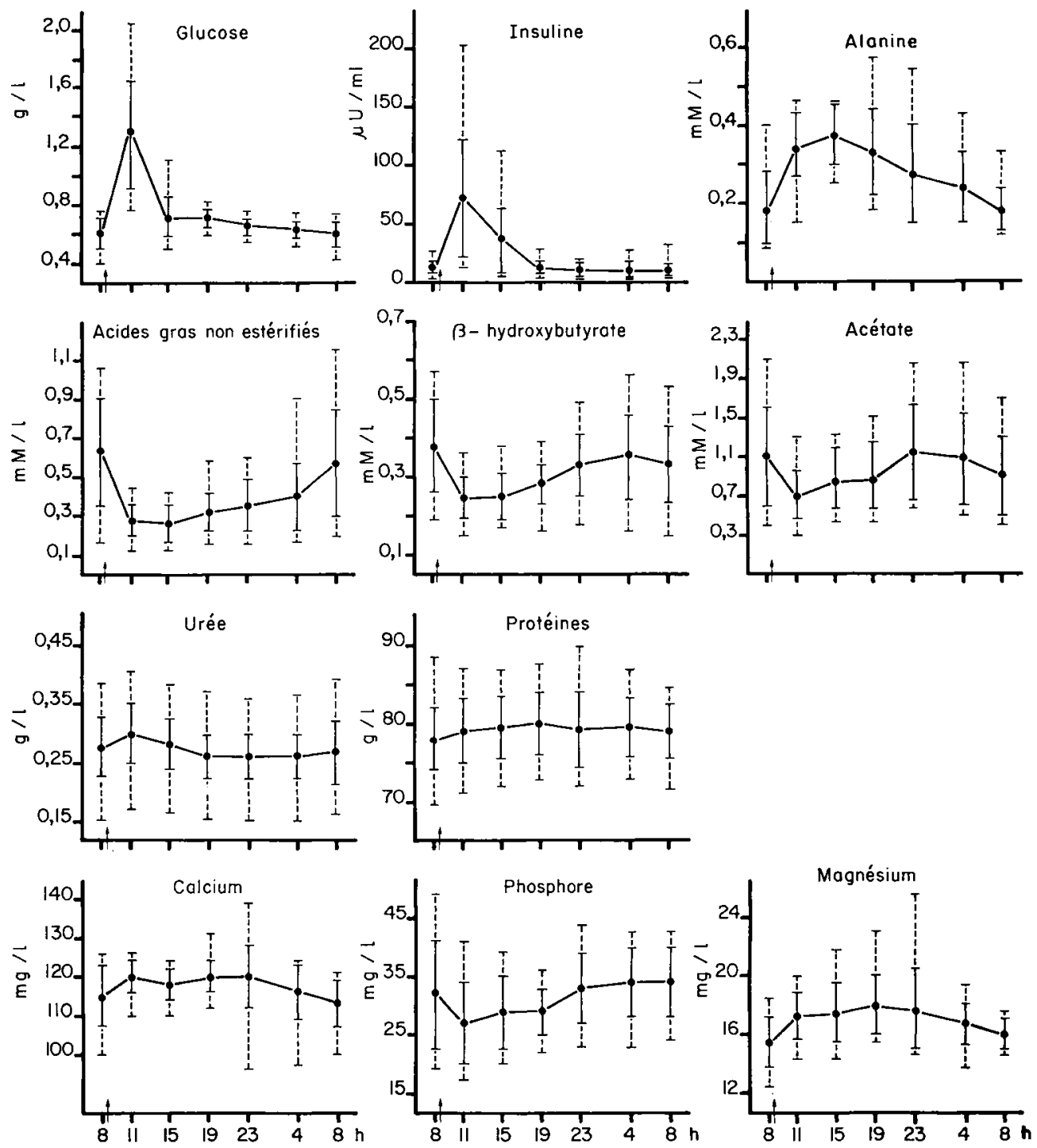

FIG. 1. - Evolution de différents constituants plasmatiques au cours de la journée (moyenne, écarts-types, limites de variation) (les écarts-types supérieur ef inférieur sont reliés par les traits pleins, les limites de variation supérieure ef inférieure par les tirets, $\uparrow$ heure de distribution des repas). 
Entre 8 et $11 \mathrm{~h}$, la calcémie et la magnésémie augmentent faiblement, respectivement de 4 et 11 p. 100, la phosphatémie diminue de 16 p. 100 . Ensuile la calcémie se stabilise puis diminue pendant la nuit; la phosphatémie suit l'évolution inverse. La magnésémie continue à augmenter au cours de la journée, atteint un maximum à $19 \mathrm{~h}$, puis diminue jusqu'au lendemain matin. Les variations au cours de la journée des teneurs individuelles sont très élevées dans le cas du phosphore : l'écart entre les concentrations extrêmes est compris entre 1 et $2 \mathrm{mg} / 100 \mathrm{ml}$ pour 18 cinétiques, soit plus de 30 p. 100 de la valeur moyenne.

Influence du régime et de l'éłat physiologique (figs $2,3,4)$. - La glycémie et l'insulinémie présentent un pic beaucoup plus marqué pour les régimes à proportion plus

1) Effet de l'état physiologique
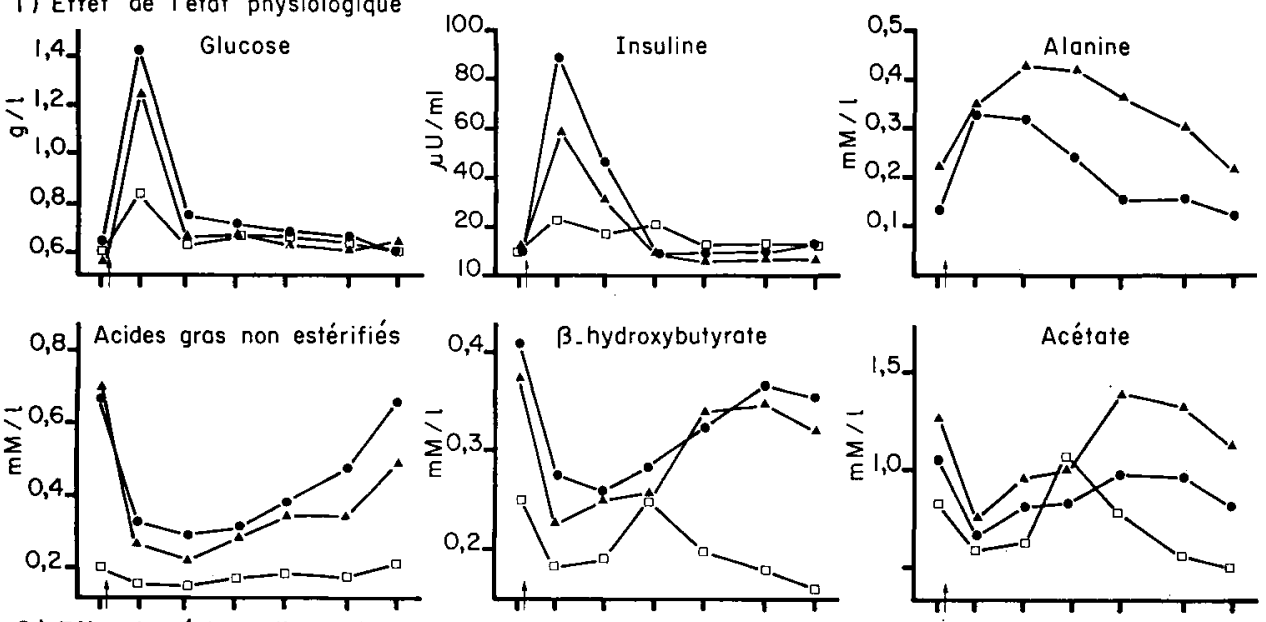

2) Effet du régime alimentaire
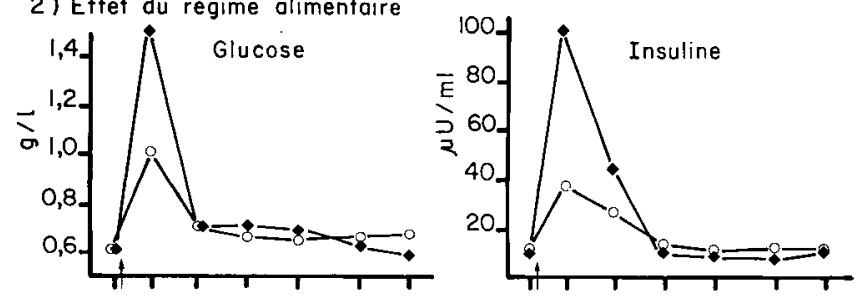

- fin de gestation

- début de lactation

- juments vides

- essai 1

- essai 2

1 heure de distribution des repas
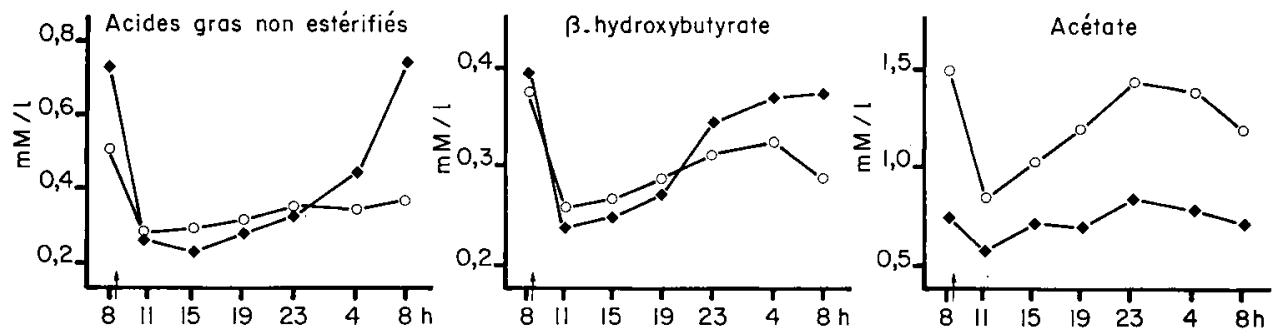

FIG. 2. - Constituants plasmatiques liés au métabolisme énergétique.

Effet de l'état physiologique et du régime alimentaire 
élevée de concentré de l'essai 2, tant en gestation qu'en lactation; l'acétate a aussi une concentration plus élevée pour ces régimes et présente des variations plus accusées avec l'heure de prélèvement. En revanche, la nature du régime n'a pas d'effet sur l'évolution des acides gras non estérifiés ef du $\beta$-hydroxybutyrate. En début de lactation, par rapport à la fin de la gestation, l'alanine diminue beaucoup plus lentement à partir de $15 \mathrm{~h}$; l'acétate, en concentration plus élevée, augmente plus rapidement au cours de la nuit. Inversement, les pics de glucose et d'insuline, de $\beta$-hydroxybutyrate et d'acides gras non estérifiés présentent une tendance non significative à des concentrations plus faibles. Les deux juments vides de l'essai 1 présentent des pics de glycémie et d'insulinémie plus faibles que les juments gestantes ou allaitantes, leurs teneurs en acides gras non estérifiés, $\beta$-hydroxybutyrate et acétate sont également plus faibles tout au long de la journée.

Le régime n'a pas d'effet significatif sur les teneurs des constifuants liés au métabolisme azoté ; cependant, se dessine une tendance à une urémie et une protéinémie plus élevées pour les régimes plus riches en azote de l'essai 1, sauf au prélèvement de $11 \mathrm{~h}$. Celles-ci sont également légèrement plus élevées en fin de gestation qu'en début de lactation; les deux juments vides présentent une urémie supérieure aux juments gestantes ou allaitantes, bien que ces différences ne soient pas significatives.

1) Effet de l'état physiologique
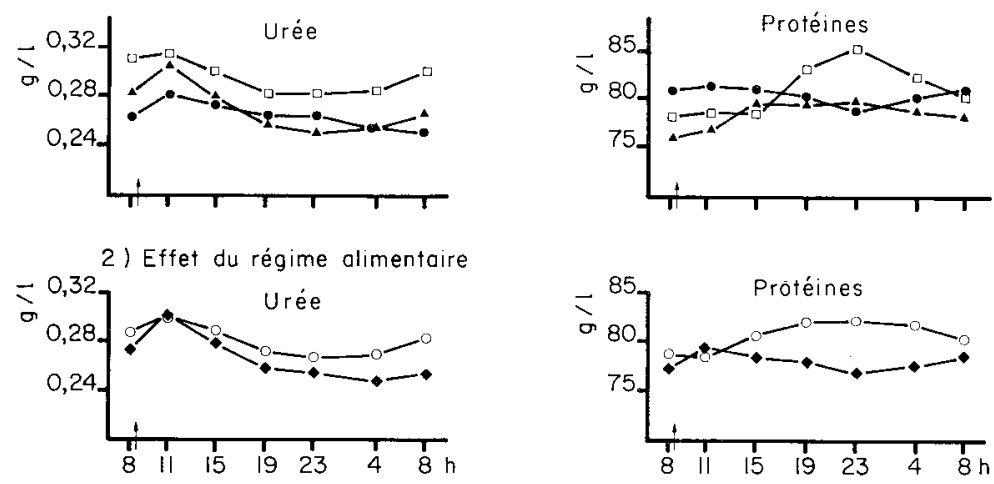

FIG. 3. - Constituants plasmatiques liés ou métabolisme azoté.

Effets de l'état physiologique ef du régime alimentaire (les symboles sont ceux de la fig. 2).

Les effets du régime et de l'état physiologique sur les teneurs en minéraux peuvent difficilement être dissociés, car les niveaux d'apport minéral dans l'aliment concentré lors de l'essai 2 sont liés à l'apport dans le fourrage, très différent entre la paille distribuée en gestation et le foin distribué en lactation. L'évolution de la calcémie ne dépend ni du régime, ni de la répartition dans la journée de l'ingestion de calcium. La phosphatémie, plus élevée dans l'essai 2, augmente rapidement entre 19 et $23 \mathrm{~h}$ pour les animaux ayant reçu un aliment concentré riche en phosphore; les phosphatémies des animaux pour lesquels le phosphore est essentiellement apporté par le foin 
augmente plus progressivement et plus tardivement. La variation de la magnésémie entre 8 et 11 h ne dépend pas de la teneur en magnésium de l'aliment concentré ; en revanche, la magnésémie augmente plus fortement au cours de la journée en début de lactation dans l'essai 2, dans lequel l'apport total de magnésium est élevé. Globalement, les teneurs en minéraux, surtout en phosphore ef magnésium, varient plus en début de lactation qu'en fin de gestation.
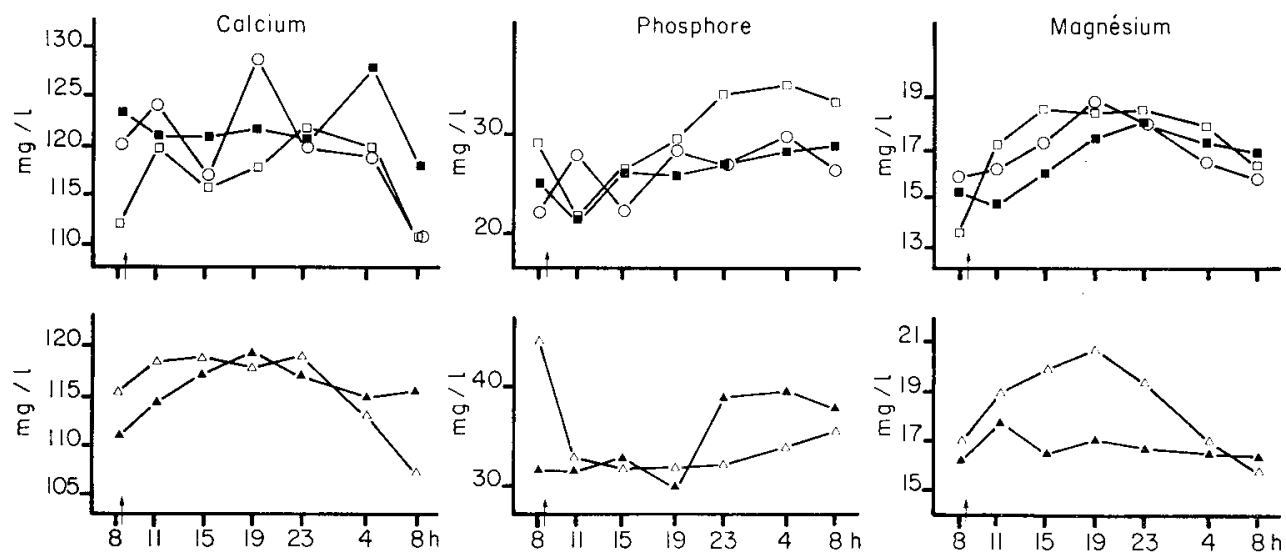

FIG. 4. - Minéraux plasmatiques. Effet de l'état physiologique et du régime alimentaire.

- Essai 1 - Fin de gestation ; $\square$ Essai 1 - Début de lactation ; O Essai 1 - Juments vides ;

$\Delta$ Essai 2 - Fin de gestation ; $\Delta$ Essai 2 - Début de lactation.

Liaisons entre différents constituants. - Afin de mettre en évidence d'éventuelles liaisons entre constituants, nous avons comparé les différences de concentration entre $8 \mathrm{~h}$ (avant la distribution d'aliments) et $11 \mathrm{~h}$ (après le repas principal). Ces différences présentent l'avantage d'être marquées pour la plupart des cinétiques. Les résultats des corrélations entre constituants liés au métabolisme énergétique ont été reportés au tableau 3. En observant séparément les résultats des essais 1 et 2, on remarque des liaisons positives hautement significatives pour les couples acides gras non estérifiésacétate, acides gras non estérifiés- $\beta$-hydroxybutyrate et acétate- $\beta$-hydroxybutyrate. Si l'on regroupe l'ensemble des cinétiques, les relations avec l'acétate ne sont plus significatives, car les pentes des régressions avec les acides gras non estérifiés et le $\beta$ hydroxybutyrate sont très différentes entre les deux essais. Une liaison positive entre les différences de teneurs en glucose et en insuline (les valeurs de l'insulinémie étant transformées en logarithme afin de leur assurer une distribution normale) s'avère significative pour l'ensemble des deux régimes, mais pas intra-régimes.

Entre 8 et $11 \mathrm{~h}$, les différences de calcémie et de magnésémie sont liées à un seuil de 1 p. $100(r=0,65)$ et les différences de phosphatémie et de magnésémie à un seuil de 5 p. $100(r=-0,47)$. En revanche, la liaison calcémie-phosphatémie est non significative $(r=-0,26)$. 
TABLEAU 3

Corrélotions entre les variations prandiales $(8 \mathrm{~h}-11 \mathrm{~h})$ des différents constituants liés au métabolisme énergétique

\begin{tabular}{|c|c|c|c|}
\hline & $\begin{array}{c}\text { Essai } 1 \\
(n=10)\end{array}$ & $\begin{array}{c}\text { Essai } 2 \\
(n=11)\end{array}$ & $\begin{array}{l}\text { Ensemble des } \\
\text { deux essais } \\
(n=21)\end{array}$ \\
\hline 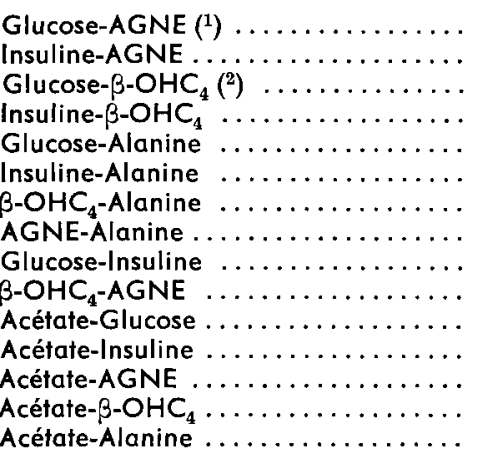 & $\begin{array}{l}-0,07 \\
-0,51 \\
-0,07 \\
-0,50 \\
= \\
= \\
\overline{-} \\
0,78 * * \\
0,87 * * \\
0,04 \\
0,50 \\
0,90 * * \\
0,86 * * \\
-\end{array}$ & $\begin{array}{l}-0,17 \\
0,32 \\
0,17 \\
0,04 \\
0,30 \\
0,26 \\
0,15 \\
-0,59 \\
0,30 \\
0,59\left({ }^{3}\right) \\
0,07 \\
-0,31 \\
0,80 * * \\
0,42\left(^{3}\right) \\
-0,32\end{array}$ & $\begin{array}{c}0,29 \\
0,38 \\
0,19 \\
0,05 \\
0,30 \\
0,26 \\
0,15 \\
-0,59 \\
0,65 * * \\
0,58 * * \\
-0,36 \\
-0,34 \\
0,21 \\
0,45 * \\
-0,32\end{array}$ \\
\hline
\end{tabular}

* Seuil de signification 5 p. 100 ; ** Seuil de signification 1 p. 100.

( $\left.{ }^{1}\right)$ AGNE : Acides gras non estérifiés.

(2) $\beta$-OHC $\mathrm{OH}_{4}: \beta$-hydroxybutyrate.

(3) Si une jument est exclue de la régression, ces corrélations sont respectivement de 0,78 ef 0,87 et significatives au seuil de 1 p. 100.

Variations individuelles. - Pour tous les constituants liés au métabolisme énergétique, sauf l'alanine, les différences individuelles les plus élevées sont observées pour les concentrations maximales ; le coefficient de variation est compris entre 25 et $70 \mathrm{p}$. 100, ce dernier chiffre étant atteint pour l'insuline. L'évolution nycthémérale des constituants présente également des variations individuelles indépendantes du régime ou de l'état physiologique. Si la glycémie est maximale à $11 \mathrm{~h}$ pour la presque totalité des cinétiques, il n'en esł pas de même pour l'insulinémie (maximum à $11 \mathrm{~h}$ et parfois à $15 \mathrm{~h}$ ), les teneurs en $\beta$-hydroxybutyrate (minimum à 11 ou $15 \mathrm{~h}$ ), en acides gras non estérifiés (minimum à 11, 15 ou $19 \mathrm{~h}$ ), en alanine (maximum à 11 ou $15 \mathrm{~h}$ ) et en acétate (minimum à $11 \mathrm{~h}$ pour la majorité des cinétiques et à des heures diverses pour les autres). II en résulte que les courbes moyennes d'évolution de certains constituants (acides gras non estérifiés, $\beta$-hydroxybutyrate, alanine) présentent un plateau de concentration entre 11 et $19 \mathrm{~h}$, alors que les courbes individuelles ne présentent pas de palier durant cette période de la journée.

Les variations individuelles de l'urémie sont très élevées quant à la valeur moyenne, mais l'évolution nycthémérale est la même pour toutes les cinétiques. En revanche, la protéinémie moyenne de la journée varie peu selon les individus.

Les variations individuelles des cinétiques de minéraux sont importantes : ainsi les évolutions entre 8 et $11 \mathrm{~h}$ sont-elles inverses de l'évolution moyenne, respectivement dans 7,5 et 4 cas sur 21 pour la calcémie, la phosphatémie et la magnésémie. 
Pour ces trois constituants, les concentrations extrêmes se situent à des heures très différentes de la journée selon les animaux, pour un même régime et un même étał physiologique.

\section{Discussion.}

Origine exogène ou endogène des variations des constituants plasmatiques.

L'évolution de la glycémie montre en premier lieu qu'une grande partie de la digestion de l'amidon de l'aliment concentré dans l'intestin grêle est déjà réalisée $2 \mathrm{~h} 30$ après sa distribution, en raison d'un transit rapide jusqu'au caecum (Alexander et Benzie, 1951 ; Argenzio ef al., 1974) dû lui-même à une motricité gastro-intestinale accrue par la prise de fourrage qui suit celle d'aliment concentré (Howell et Cupps, 1950). Cet apport massif de glucose entraîne une augmentation de glycémie beaucoup plus importante que celle relevée antérieurement par Takagi et Sakurai (1961), Gill, Skwarlo et Flisinska-Bojanowska (1974), Evans, Thompson et Wingef (1974) ef Anderson (1975) ; la comparaison avec ces travaux esi cependant difficile, car aucun de ces auteurs n'a précisé l'alimentation des chevaux utilisés. Seuls Camus (1972) ef Wolter et al. (1978) notent, respectivement à la suite d'ingestion d'avoine ef d'aliment complet, une augmentation de glycémie presque aussi importante. L'augmentation de la glycémie est de courte durée dans notre essai, puisqu'elle est revenue à la valeur pré-prandiale 6 h 30 après le repas d'aliment concentré. La digestion de l'amidon d'une part et l'utilisation du glucose formé de l'autre sont donc très rapides ; ce dernier aspect est à rapprocher de tests de tolérance à l'ingestion de sucres (Roberts, 1975) et à l'injection de glucose (Mehring et Tyznik, 1970) qui avaient mis en évidence des résultats comparables. L'évolution de la glycémie chez le cheval après le repas se rapproche plus de celle du veau préruminant (Thivend et Martin-Rosset, 1971) que du porc (Marshall ef al., 1973) ou de l'homme (Tasaka ef al., 1975), chez qui l'augmentation post-prandiale est plus faible et plus étalée, probablement en raison d'une période d'absorption plus longue, $6 \mathrm{~h}$ au moins chez le porc (Rérat, Vaissade et Vaugelade, 1979). L'augmentation de l'insulinémie est concomitante à celle du glucose, ainsi que l'ont montré Argenzio et Hintz (1971) ; ces mêmes auteurs ont d'ailleurs relié la production d'insuline à l'apport de glucose (Argenzio et Hintz, 1972). Le retour tardif à l'insulinémie pré-prandiale, ef le fait qu'elle est parfois plus élevée 6 h 30 que 2 h 30 après la distribution d'aliments, confirment que la production et la vitesse de disparition de l'insuline chez le cheval sont relativement lentes (Madigan et Evans, 1973). Par ailleurs nous n'observons pas le changement de l'insulinémie ef de la glycémie avec l'éclairement, constaté par Evans, Thompson et Winget (1974).

Les variations nycthémérales de la concentration en acides gras volatils dans le gros intestin du cheval, étudiées notamment par Stillions, Teeter et Nelson (1970), Argenzio, Southworth et Stevens (1974,) Tisserand et al. (1977), Wolter et al. (1978) et Schwabenbauer (1979), montrent un pic de concentration peu marqué en propionate et plus important en acétate 6 à $12 \mathrm{~h}$ après le repas, mais pas de pic de butyrate. L'absence d'effet de la production de propionate sur la glycémie est à relier aux observations d'Argenzio ef Hintz (1970) : une injection de propionate dans le sang ne provoque d'augmentation de la glycémie qu'en période de jeûne ; il est par ailleurs 
possible que dans notre essai il n'y ait pas de pic de production de propionate, en raison d'une digestion complète de l'aliment concentré dans l'intestin grêle ou d'une ingestion de fourrage très étalée. Chez le cheval, l'acétate et le $\beta$-hydroxybutyrate plasmatiques proviennent en partie, comme chez le ruminant, de l'acétate et du butyrate produits par la dégradation microbienne des glucides. L'accroissement nocturne d'acétate plasmatique que nous avons observé correspond au pic de concentration en acétate dans le gros intestin. Le butyrate, produit sans grandes variations de concentration au cours de la journée, serait, d'après Giddings et Stevens (1968) et Lieb, Baker et Crawford (1970) converti en 3 -hydroxybutyrate non au niveau de la paroi du tractus digestif comme le ruminant, mais essentiellement au niveau du foie. Il est possible que, comme chez le rat, cette transformation soit d'autant plus forte que l'animal est à jeûn (Rémésy, Demigné et Chartier, 1980) ce qui pourrait contribuer à l'accroissement nocturne du $\beta$-hydroxybutyrate plasmatique.

L'évolution nycthémérale de la teneur en acides gras non estérifiés traduit l'alternance entre d'une part une phase de mobilisation des réserves lipidiques corporelles en période nocturne, lorsque les activités alimentaires sont les plus faibles, et une phase d'arrêt de la lipolyse et d'utilisation des acides gras non estérifiés par les tissus périphériques, qui se manifeste au moment de l'ingestion du repas d'aliment concentré. Les variations d'acétate et de $\beta$-hydroxybutyrate sont fortement liées à celles des acides gras non estérifiés et peuvent être interprétées de la même manière. Il est à remarquer que la diminution rapide de ces trois constituants coïncide avec le pic d'insuline, hormone antilipolytique favorisant l'utilisation des substrats énergétiques par les tissus périphériques. La possibilité pour le cheval de mobiliser rapidement ses lipides corporels, à l'échelle d'un nycthémère, est un phénomène assez général tant chez les ruminants comme la vache (Coggins ef Field, 1976) que chez les monogastriques comme le rat (Phan, Bach et Metais, 1974) ; elle avait déjà été signalée chez le cheval par Delamarre (1974). L'augmentation post-prandiale de la teneur en alanine n'est probablement due qu'en partie à l'absorption accrue au niveau de l'intestin grêle ; elle doit être également liée à l'arrêt de la néoglucogenèse, consécutif à un apport accru de glucose et à un effet inhibiteur de l'insuline. La diminution de l'alaninémie à partir du soir peut être attribuée à une reprise de la néoglucogenèse.

Chez le ruminant, l'urémie est fortement liée aux concentrations en ammoniac dans le rumen. Chez le cheval, les données relatives à l'évolution nycthémérale de la concentration en ammoniac dans le cæcum diffèrent selon les auteurs. Si d'après Candau ef Vigroux (1974) puis Schwabenbauer (1979), le maximum se situe $2 \mathrm{~h}$ après le repas, il n'en est pas de même pour Wootton et Argenzio (1975), Tisserand et al. (1977) et Wolter et al. (1978) qui constatent des maxima plus éloignés du repas. Ces divergences doivent provenir en partie de variations du temps de transit des digesta jusqu'au cæcum ; il est de toute manière probable que la concentration moyenne en ammoniac de l'ensemble du gros intestin (le cæcum en représentant moins d'un quart, Wolter et Gouy, 1976) ne varie pas de la même manière, l'ammoniac formé provenant soit de la dégradation des protéines alimentaires ou endogènes, soit de l'autolyse des protéines microbiennes. Il est cependant possible que dans notre essai, la modeste mais systématique augmentation de l'urémie 2 h 30 après le repas soit liée à un accroissement de la teneur en ammoniac du gros intestin ; elle pourrait plus probablement, de même que chez la plupart des monogastriques, être due à des désaminations 
dans le foie lors du pic d'absorption d'acides aminés, accompagnées d'une augmentation d'activité des enzymes du cycle de l'urée, comme l'ont montré Kato ef al. (1978) chez le rat. Il est également possible que l'augmentation de l'urémie dans notre essai soit liée à l'absorption au niveau de l'intestin grêle d'urée provenant de la salive, puisque celle-ci est émise en quantité importante, en particulier au moment des repas (Colin, 1871).

L'évolution des teneurs en minéraux au cours de la journée à principalement été étudiée chez le rat. Chez celui-ci, alors qu'il consomme ses aliments uniquement en période d'obscurité, la calcémie et la phosphatémie sont maximales $3 \mathrm{~h}$ après la fin d'une période d'obscurité (Milhaud, Pérault-Staub et Staub, 1972), alors que la magnésémie s'élève pendant les 6 premières heures de la prise alimentaire puis revient au niveau initial pour rester stable pendant la période d'éclairement (Rayssiguier ef Larvor, 1974). Les variations nycthémérales sont beaucoup moins nettes chez la jument qui répartit mieux ses activités alimentaires entre le jour et la nuit (Doreau, 1978). Cependant, on peut associer la légère élévation de la calcémie et de la magnésémie ef la diminution de la phosphatémie faisant suite à l'ingestion d'aliment concentré à l'absorption du calcium ef du magnésium qui s'effectue principalement dans l'intestin grêle (Schryver et al., 1970 ; Hintz et Schryver, 1972) ef à une résorption nette de phosphore à ce niveau, due à l'absence d'absorption et à la sécrétion d'ions phosphate dans l'intestin grêle lors du passage des digesta (Schryver et al., 1972). La calcémie se stabilise jusqu'au soir, sans doute en raison de l'étalement de l'absorption de calcium présent en quantités importantes dans les fourrages (fig. 1). De même, l'augmentation constante de la magnésémie jusqu'au soir traduit peut-être la richesse de la ration en magnésium.

\section{Influence du régime et de l'état physiologique.}

Les pics de glycémie sont plus accentués lorsque la proportion d'aliment concentré dans la ration s'accroît. Ce résultat est à rapprocher des observations d'Aumaitre ef al. (1973) chez le porc. Inversement Hintz, Argenzio et Schryver (1971) puis Ralston, Van den Broek et Baile (1979) ont constaté que l'augmentation de glycémie était indépendante du rapport fourrage/concentré ou de l'apport de glucides non pariétaux. Cette contradiction pourrait provenir du niveau des apports et des besoins des animaux : ces auteurs ont utilisé des poneys à l'entretien et l'apport de glucose restait très modeste. L'état physiologique n'a pas d'effet sur la glycémie et l'insulinémie de base ; selon Evans (1971) il n'y a pas de liaison entre la glycémie plasmatique et la vitesse d'utilisation du glucose, malgré des besoins très importants en lactation (Anwer et al., 1975), alors que le jeûne provoque une diminution de glycémie. Il semblerait ainsi qu'il faille une sous-nutrition énergétique très intense pour que la glycémie soit affectée chez le cheval. Les teneurs pré-prandiales en acides gras non estérifiés et, à un degré moindre, en $\beta$-hydroxybutyrate, sonł plus élevées avec le régime apportant globalement moins d'énergie ; cette constatation, qui reste à confirmer sur un nombre plus élevé de données, permet de considérer les teneurs préprandiales en ces deux constituants comme des indices de sous-nutrition énergétique, de même que chez le ruminant (Decaen et Journet, 1967 ; Bowden, 1971). De même, les faibles teneurs en acides gras non estérifiés et en $\beta$-hydroxybutyrate des juments 
vides sont à rapprocher du niveau élevé de couverture des besoins énergétiques. La différence minime des teneurs en fin de gestation et en début de lactation permet de penser que les apports énergétiques plus élevés en début de lactation ont correspondu à l'accroissement des besoins. La teneur en acétate est beaucoup plus élevée pour les régimes plus riches en fourrage : les fortes variations de l'acétate plasmatique avec le rapport fourrage/concentré avaient déjà été mises en évidence par Jean-Blain (1973). La teneur en acétate est plus élevée et ses variations plus marquées en débuł de lactation alors que l'accroissement des apports exogènes d'acétate aurait dû être compensé par une utilisation plus importante comme précurseur des acides gras du lait : il est possible qu'il y ait en début de lactation une limitation de l'utilisation de l'acéfate par insuffisance des disponibilités en glucose, tant d'origine exogène qu'endogène. En effet, la néoglucogenèse semble moins importante en début de lactation qu'en fin de gestation, comme en témoigne la diminution lente de l'alaninémie.

L'urémie présente des valeurs légèrement plus élevées avec le régime apportant le plus d'azote, ainsi que pour les juments vides à faibles besoins. Les différences sont cependant minimes : en effet, la plage de variation de l'urémie chez le cheval est importante (Bost et al., 1970).

La phosphatémie et la magnésémie dépendent du régime, mais pas la calcémie ; ceci provient peut-être du niveau de couverture des besoins, et d'une meilleure régulation de la calcémie. La magnésémie plus élevée en début de lactation, alors que les besoins sont plus importants qu'en fin de gestation, n'est que partiellement explicable par les interactions entre les apports nutritifs et le stade physiologique. ll semble, d'une manière générale, que la régulation des teneurs en éléments minéraux soit plus efficace en fin de gestation qu'en début de lactation.

\section{Conclusion.}

Ces cinétiques nycthémérales des constituants plasmatiques chez le cheval ont permis de mettre en évidence :

10 l'origine endogène et exogène des variations. Le déroulement de la digestion chez le cheval étant plus proche de celui du monogastrique non herbivore que du ruminant, les évolutions nycthémérales sont en général comparables à celles d'un monogastrique. Cependant, il arrive que les variations d'origine endogène et exogène se superposent : c'est le cas du $\beta$-hydroxybutyrate. Ce dernier est cependant apparu comme un témoin d'une sous-nutrition énergétique d'emploi plus facile que chez le ruminant;

$2^{\circ}$ l'amplitude considérable de variation des constituants plasmatiques selon les individus ou l'heure de prélèvement. La réalisation de profils métaboliques nécessitera donc certaines précautions. L'heure du prélèvement par rapport à la prise de nourriture sera déterminée en fonction de l'objectif recherché et des constituants étudiés. Pour mettre en évidence des différences entre régimes, il sera préférable d'effectuer le prélèvement sanguin lorsque la variation du ou des constituants est maximale ; au contraire, pour caractériser un régime ou l'état nutritionnel d'un troupeau, il conviendra de l'effectuer lorsque la variation est minimale.

Reçu en avril 1980. Accepté en juillet 1980. 
Remerciements. - Nous tenons à remercier tous ceux qui nous ont aidés dans ce travail pour leur aide matérielle et leurs conseils, en parriculier, J. Espinasse, R. Espinasse,

J. Grizard, M. Journet, C. Rémésy.

\section{Références}

ALEXANDER F., BENZIE D., 1951. A radiological study of the digestive tract of the foal. Quart. J. exp. Physiol., 36, 213-217.

ANDERSON M. G., 1975. The effect of exercise on blood metabolite levels in the horse. Equine vet. J., 7, 27-33.

ANWER M. S., GRONWALL R., CHAPMAN J. E., KLENTZ R. D., 1975. Glucose utilization and contribution to milk components in lactating ponies. J. onim. Sci., 41, 568-571.

ARGENZIO R. A., HINTZ H. F., 1970. Glucose tolerance and effect of volatile fatty acid on plasma glucose concentration in ponies. J. anim. Sci., 30, 514-518.

ARGENZIO R. A., HINTZ H. F., 1971. Volatile fatty acid tolerance and effect of glucose and VFA on plasma insulin levels in ponies. J. Nutr., 101, 723-730.

ARGENZIO R. A., HINTZ H. F., 1972. Effect of diet on glucose entry and oxidation rates in ponies. J. Nutr., 102, 879-892.

ARGENZIO R. A., LOWE J. E., PICKARD D. W., STEVENS C. E., 1974. Digesta passage and water exchange in the equine large intestine. Am. J. Physiol., 226, 1035-1042.

ARGENZIO R. A., SOUTHWORTH M., STEVENS C. E., 1974. Sites of organic acid production and absorption in the equine gastrointestinal tract. Am. J. Physiol., 226, 1043-1050.

AUMAITRE A., RÉRAT A., VAISSADE P., VAUGELADE P., 1973. Etude expérimentale qualitative de l'absorption infestinale des glucides après ingestion d'un repas à base de glucose ou d'amidon. Ann. Biol. anim. Bioch. Biophys., 13, 784-788.

BOST J., FONTAINE M., JEAN-BLAIN C., LAPRAS M., MAGAT A., 1970. Evaluation de certains constituants du sang chez les chevaux cliniquement normaux. Ann. Rech. véfer., 1, 63-91.

BOWDEN D. M., 1971. Non-esterified fatty acids and ketone bodies in blood as indicators of nutritional status in ruminants : a review. Con. J. anim. Sci., 51, 1-13.

CAMUS E., 1972. Variation de la glycémie chez le cheval. Th. ENV Lyon, 50 pp.

CANDAU M., VIGROUX P., 1974. Sur le mécanisme de l'hypermotilité cæcale liée au repas chez le cheval. C. R. Soc. Biol., 168, 893-897.

COGGINS C. R. E., FIELD A. C., 1976. Diurnal variation in the chemical composition of plasma from lactating beef cows on three diefary energy intakes J. agric. Sci., Camb., 86, 595-602.

COLIN G., 1871. Traité de physiologie comparée des animaux, 600-615, $2^{\mathrm{e}}$ édit., Baillière, Paris.

DECAEN C., JOURNET M., 1967. Evolution au début de la lactation de la sécrétion des principaux acides gras du lait et de la concentration en acides gras libres du sang chez la vache. Ann. Biol. anim. Bioch. Biophys., 7, 131-143.

DELAMARRE H., 1974. Quelques aspects de l'adaptation métabolique du cheval à l'inanition. Th. Doct.Vét., 98 pp., Univ. Claude-Bernard, Lyon.

DOREAU M., 1978. Comportement alimentaire du cheval à l'écurie. Ann. Zoofech., 27, 291-302.

ERRINGTON B. J., 1937. Variations in inorganic phosphorus and calcium content of the blood of horses. Cornell Vef., 27, 1-13.

EVANS J. W., 1971. Effect of fasting, gestation, lactation and exercise on glucose turnover in horses. J. anim. Sci., 33, 1001-1004.

EVANS J. W., THOMPSON P. G., WINGET C. M., 1974. Glucose and insulin biorhythms in the horse. J. S. Afr. Vet. Assoc., 45, 317-329.

GIDDINGS R. F., STEVENS C. E., 1968. In vitro studies of electrolyte and fatty acid transport across the epithelium of the horse cecum. Proc. 1st Eq. Nutr. Symp., 15-16.

GILL J., SKWARLO K., FLISINSKA-BOJANOWSKA A., 1974. Diurnal and seasonal changes in carbohydrate metabolism in the blood of thoroughbred horses. J. inferdiscipl. Cycle Res., 5, 355-361.

GUYNN R. W., VEECH R. L., 1975. Enzymatic determination of acetate, 302-307. In LOWENSTEIN J. M., Methods in enzymology, Vol. 35, Part. B. Acad. Press, New York. 
HALES C. N., RANDLE P. J., 1963. Immunoassay of insulin with insulin-antibody precipitate. Biochem. J., 88, 137.146.

HINTZ H. F., ARGENZIO R. A., SCHRYVER H. F., 1971. Digestion coefficients, blood glucose levels and molar percentage of volatile acids in infestinal fluid of ponies fed varying forage-grain ratios. J. anim. Sci., 33, 992-995.

HINTZ H. F., SCHRYVER H. F., 1972. Magnesium metabolism in the horse. J. anim. Sci., 35, 755-759. HOWELL C. E., CUPPS P. T., 1950. Motility patterns of the cecum of the horse. J. anim. Sci., 9, 261-268.

JEAN-BLAIN C., 1973. Variations de la teneur en acides gras volatils (AGV) du sang périphérique, chez le cheval, en fonction du régime alimentaire et de l'activité musculaire. Ann. Biol. anim. Bioch. Biophys., 13, 763-765.

JOHNSON R. J., HART J. W., 1974. Influence of feeding and fasting on plasma free amino-acids in the equine. J. onim. Sci., 38, 790-794.

KATO H., MIZUTANI-FUNAHASHI M., SHIOSAKA S., NAKAGAWA H., 1978. Circadian rhythms of urea formation and arginosuccinate synthetase activity in rat liver. J. Nutr., 108, 1071-1077.

KRZYWANEK H., 1969. Die Konzentration freier Fettsäuren in Blutplasma trainierter Trabrennpferde. Z. Tierphysiol. Tierernähr. Futtermittelk., 25, 236-238.

LIEB S., BAKER J. P., CRAWFORD B. H. Jr., 1970. Energy absorption and utilization in the equine. J. anim. Sci., 31, 207-208.

MADIGAN J. E., EVANS J. W., 1973. Insulin furnover and irreversible loss rate in horses. J. onim. SCi., 36, 730-733.

MARSHALL M., SCHUCKERT G., SPRANDEL U., ZÖLLNER N., 1973. Zirkadiane Schwankungen von Plasmaglucose und Insulin beim jungen Miniaturschwein. Zbl. Vet. Med. A, 20, 382-387.

MEHRING J. S., TYZNIK W. J., 1970. Equine glucose tolreance. J. anim. Sci., 30, 764-766.

MILHAUD G., PERAULT-STAUB A. M., STAUB J. B., 1972. Diurnal variation of plasma calcium and calcitonin function in the rat. J. Physiol., 222, 559-567.

MOORE J. J., SAX S. M., 1965. A revised aufomated procedure for urea nitrogen. Clin. chim. Acfo, 11, 475-476.

PHAN T., BACH A., METAIS P., 1974. Effects of fasting on intermediate hepatic metabolism of the rat. Arch. int. Physiol. Biochim., 82, 603-609.

RALSTON S. L., VAN DEN BROEK G., BAILE C. A., 1979. Feed intake patterns and associated blood glucose, free fatty acid and insulin changes in ponies. J. anim. Sci., 49, 838-845.

RAYSSIGUIER Y., LARVOR P., 1974. Variations nycthémérales de la magnésémie chez le rat : influence du jeûne et de la réplétion stomacale. Ann. Biol. anim. Bioch. Biophys., 14, 821-827.

RÉMÉSY C., DEMIGNÉ C., 1976. Variations in some plasma metabolites from neoglucogenesis and ketogenesis in pregnant ewes in relation to diet. Ann. Rech. vétér. 7, 329-341.

RÉMÉSY C., DEMIGNÉ C., CHARTIER F., 1980. Origin and utilization of volatile fatty acids in the rat. Reprod. Nutr. Dévelop., 20, 1339-1349.

RÉRAT A., VAISSADE P., VAUGELADE P., 1979. Absorption kinetics of amino acids and reducing sugars during digestion of barley or wheat meals in the pig : preliminary data. Ann. Biol. anim. Bioch. Biophys., 19, 739-747.

ROBERTS M. C., 1975. Carbohydrate digestion and absorption studies in the horse. Res. vet. Sci., 18, 64-69.

RUCKEBUSCH Y., VIGROUX P., CANDAU M., 1976. Analyse du comportement alimentaire chez les Equidés. C. R. Journ. Ełud. CEREOPA, 69-72.

SCHRYVER H. F., CRAIG P. H., HINTZ H. F., HOGUE D. E., LOWE J. E., 1970. The site of calcium absorption in the horse. J. Nutr., 100, 1127-1132.

SCHRYVER H. F., HINTZ H. F., CRAIG P. H., HOGUE D. E., LOWE J. E., 1972. Site of phosphorus absorption from the intestine of the horse. J. Nutr., 102, 143-148.

SCHWABENBAUER K., 1979. Einfluss von Futferzusammensetzung und Fütferungstechnik auf mikrobielle Umsetzungen in Blinddarm des Pferdes. Inaug. Diss. Doct. Med. Vet., 117 pp., Hannover.

STILLIONS M. C., TEETER S. M., NELSON W. E., 1970. Equine digestive volatile fatty acid concentration. Prac. 2nd Eq. Nutr. Res. Symp., 21-22.

TAKAGI S., SAKURAI N., 1971. Changes of glucose, pyruvate and lactate in blood of horses at rest and during exercise. Exp. Rep. Equine Hlth. Lab., 8, 100-109. 
TASAKA Y., SEKINE M., WAKATSUKI M., OHGAWARA H., SHIZUME K., 1975. Levels of pancreatic glucagon, insulin and glucose during twenty-four hours of the day in normal subjects. Horm. Metab. Res., 7, 205-206.

THIVEND P., MARTIN-ROSSET W., 1971. Etude des variations post-prandiales de la glycémie mésentérique chez le veau préruminant. Ann. Biol. anim. Bioch. Biophys., 11, 350-351.

TISSERAND J. L., CANDAU M., HOUISTE A., MASSON C., 1977. Evolution de quelques paramètres physico-chimiques du contenu cæcal d'un poney au cours du nycthémère. Ann. Zootech., 26, 429-434.

TRINDER P., 1969. Determination of glucose in blood using glucose oxydase with an alternative oxygen acceptor. Ann. clin. Biochem., 6, 24-27.

WILLIAMSON D. H., 1974. L-alanine. Determination with alanine dehydrogenase, 1679-1682. In BERGMEYER H. U. Methods of enzymatic analysis, Vol. 4, Acad. Press, New York.

WILLIAMSON D. H., MELLANBY J., 1974. D- $(-) \beta$-hydroxybutyrate, 1836-1839. In BERGMEYER H. U. Methods of enzymatic analysis, Vol. 4, Acad. Press, New York.

WOLTER R., GOUY D., 1976. Etude expérimentale de la digestion chez les équidés par analyse du contenu intestinal après abattage. Rev. Méd. vét., 127, 1723-1736.

WOLTER R., GOUY D., DURIX A., LETOURNEAU J. C., CARCELEN M., LANDREAU J., 1978. Digestibilité et activité biochimique intracæcale chez le poney recevant un même aliment complet présenté sous forme granulée, expansée ou semi-expansée. Ann. Zoofech., 27, 47-60.

WOOTTON J. F., ARGENZIO R. A., 1975. Nitrogen utilization within equine large intestine. Am. J. Physiol., 229, 1062-1067. 